\title{
Effects of PKM2 Gene Silencing on the Proliferation and Apoptosis of Colorectal Cancer LS-147T and SW620 Cells
}

\author{
Ran Ao Lin Guan Ying Wang Jia-Ni Wang \\ Department of Gastroenterology, the First Affiliated Hospital of CMU, Shenyang, P.R. China
}

\section{Key Words}

Pyruvate kinase M2 • Colorectal cancer • Apoptosis • Proliferation • LS-147T • SW620

\begin{abstract}
Background/Aims: This paper aims to explore the effects of pyruvate kinase (PK) M2 gene silencing on the proliferation and apoptosis of colorectal cancer (CRC) LS-147T and SW620 cells. Methods: CRC LS-147T and SW620 cells highly expressing PKM2 were randomly selected by quantitative real-time polymerase chain reaction (qRT-PCR) and then assigned into the blank (no transfection), PKM2-shRNA (transfection with shRNA) and empty plasmid (transfection with empty plasmid) groups. Immunofluorescence was applied to detect PKM2 protein expression. qRT-PCR and Western blotting were conducted to assess mRNA and protein expression of $P K M 2, p 53$ and $p 21$. The cell counting kit-8 (CCK-8) assay was used to assess cell proliferation. Flow cytometry was used to assess the cell cycle and apoptosis rate, and a senescence-associated $\beta$-galactosidase staining kit was used to assess cell senescence. Results: PKM2 exhibited high mRNA expression among CRC LS-147T and SW620 cells with remarkable protein expression noted in the cytoplasm and nucleus. The PKM2-shRNA group exhibited reduced PKM2 mRNA and protein expression, whereas $p 53$ and $p 21$ expression was increased compared with the blank and empty plasmid groups. Cell proliferation in PKM2shRNA cells decreased significantly compared with the blank group and empty plasmid groups. The PKM2-shRNA group exhibited more cells in the $G_{1}$ phase and fewer cells in the $G_{2} / M$ phase compared with the blank and empty plasmid groups. In addition, the PKM2-shRNA group exhibited significantly increased apoptosis rates and $\beta$-galactosidase activity compared with the blank and empty plasmid groups. Conclusion: Our study demonstrates that PKM2 gene silencing suppresses proliferation and promotes apoptosis in LS-147T and SW620 cells.
\end{abstract}

\section{Introduction}

Colorectal carcinoma (CRC), also known as rectal carcinoma and including colon cancer and colorectal cancer, is a prevalent gastrointestinal malignant tumor [1]. CRC presents critical threats to human health, as it is the second-most diagnosed among female malignant tumors and third-most among males [2]. Modern medicine revealed that CRC occurs in the large intestine mucosal epithelium and is initiated by diverse carcinogens as well as genetic

Dr. Ran Ao

KARGER
Department of Gastroenterology, the First Affiliated Hospital of CMU, No. 155, North Nanjing Street, Heping District, Shenyang 110001, Liaoning Province, (P.R. China) Tel. +86-024-83283720, E-Mail kiss_aoran@126.com 


\section{Cellular Physiology Cell Physiol Biochem 2017;42:1769-1778 \\ \begin{tabular}{l|l} 
DOI: 10.1159/000479456 & O 2017 The Author(s). Published by S. Karger AG, Basel \\
www.karger.com/cpb
\end{tabular} \\ Ao et al.: PKM2 and LS-147T and SW620 Cell Apoptosis and Proliferation}

and environmental factors [3]. Early diagnosis of CRC is highly curative (90\% cure rate), whereas the 5-year survival rate of progressive CRC (metastasis occurring) remains less than $10 \%$, resulting from the fact that CRC has occult pathogenesis in early stages and is typically diagnosed in the progression stage, which poses difficulties for therapy [4, 5]. Although surgery is the preferred CRC treatment, statistics indicate that the 5-year survival rate of the postoperative period remained only approximately $50 \%$, and the 30 -d mortality following surgery reached 3\% to $10 \%$ [6]. Additionally, patients in various stages of treatments typically exhibited varied symptoms, such as cancer-related fatigue (CRF) induced by the disease itself and the therapeutic regimen [7]. With an increasing number of further explorations of CRC pathogenesis, pyruvate kinase M2 (PKM2), which promotes tumor cell proliferation and migration, has increasingly become the focus of multiple malignant tumor studies [8], and an increasing number of researchers began to perform specific PKM2 carcinogenesis investigations to improve CRC treatments.

Pyruvate kinase (PK), which is a key enzyme in glycolysis, catalyzes phosphoenolpyruvate into pyruvate and releases energy [9]. There are 4 subtypes of PK, including PKL, PKR, PKM1 and PKM2 (splice variant of PKM1) [10]. Among them, human PKM2 is expressed in fetal tissues and is gradually replaced by another three isoenzymes after birth. Moreover, PKM2 exhibits high expression in tumor tissues and plays important roles in growth, proliferation and the migration of tumor cells [11]. Furthermore, PKM2 is associated with numerous neoplastic diseases, such as human glioblastoma [12], prostate cancer [13], breast cancer [14] and cholangiocarcinoma [15], whereas its definite pathogenesis remains unclear. RNA interference (RNA) triggered by double-stranded RNA is characterized by precise sequence specificity and efficiency, and RNA therapy is evolving as a promising player in the battle against cancer, given its post-transcriptional gene silencing ability [16]. Dysregulated metabolic pathways, which are common in different cancer cells, are one of the significant hallmarks of cancer [17]. Increased miR-144 suppresses proliferation and improves apoptosis and autophagy in lung cancer cell lines (H460 and A549 cells) [18]. Therefore, RNA gene silencing was introduced in this article to establish a PKM2-silencing system in CRC LS147T and SW620 cell lines and analyze its effect on LS-147T and SW620 cell apoptosis and proliferation. We sought to assess whether PMK2 can be considered a marker and clinical therapeutic target for the early diagnosis of CRC.

\section{Materials and Methods}

\section{Cell Culture}

Human CRC LS-147T, HT29, DLD1, HCT116, SW620 and SW480 cell lines (provided by the Molecular Biology Laboratory of Shandong University, Shandong, China) were inoculated in RPMI 1640 medium (Solarbio, Shanghai, China) containing 10\% fetal bovine serum (FBS) (Solarbio, Shanghai, China). Cells were cultured in a $37^{\circ} \mathrm{C}$ sterile incubator with $5 \% \mathrm{CO}_{2}$. Cell passage was conducted with $0.25 \%$ trypsin digestion (Solarbio, Shanghai, China) when cells were completely adherent to the culture flask. Cells in the logarithmic phase were collected for subsequent experiments.

\section{Quantitative Real-time Polymerase Chain Reaction ( $q$ RT-PCR)}

The total RNA was extracted using a kit (Solarbio, Shanghai, China), and then the 0D260/280 of the RNA sample was detected by ultraviolet spectrophotometery. In addition, the RNA concentration was measured. The sample was then stored at $-80^{\circ} \mathrm{C}$ for further use. Based on the Genebank data for primer sequences, Primer 5.0 software was used for PKM2 primer sequences (Invitrogen Inc., Shanghai, China) (Table 1). Reverse transcription was conducted following the instructions of Reverse Transcription System A3500 (Promega Corporation, Madison, WI, USA). Reaction conditions were as follows: initial denaturation at $95^{\circ} \mathrm{C}$ for $3 \mathrm{~min} ; 40$ cycles of denaturation at $95^{\circ} \mathrm{C}$ for $30 \mathrm{~s}$, annealing at $55^{\circ} \mathrm{C}$ for $30 \mathrm{~s}$, extension at $72^{\circ} \mathrm{C}$ for $1 \mathrm{~min}$; and finally $72^{\circ} \mathrm{C}$ for $5 \mathrm{~min}$. The reaction system contained $12.5 \mu \mathrm{L}$ of Premix Ex Taq or SYBR Green Mix, $1 \mu \mathrm{L}$ of Forward Primer, $1 \mu \mathrm{L}$ of Reverse Primer, $4 \mu \mathrm{L}$ of DNA template and $25 \mu \mathrm{L}$ of ddH $\mathrm{O}_{2}$. Using $G A P D H$ as the internal reference, a solubility curve was used to detect PCR reliability and primer specificity. 


\section{Cellular Physiology Cell Physiol Biochem 2017;42:1769-1778 \begin{tabular}{l|l|l} 
and Biochem 10.1159/000479456 & $\begin{array}{l}\text { C } 2017 \text { The Author(s). Published by S. Karger AG, Basel } \\
\text { www.karger.com/cpb }\end{array}$
\end{tabular} \\ Ao et al.: PKM2 and LS-147T and SW620 Cell Apoptosis and Proliferation}

The $2^{-\Delta \triangle \mathrm{Ct}}$ method was employed to assess PKM2 mRNA expression with a multiplication rate equal to the relative expression of the target gene with $\Delta \mathrm{Ct}=\mathrm{Ct}_{\text {target gene }}-\mathrm{Ct}_{\text {internal reference gene }}[19]$. This method was also adapted for the expression of PKM2, p53 and p21 after transfection.

Construction of the PKM2 shRNA Lentivirus

According to Invitrogen, the PKM2 shRNA sequence was 5 '-CATCTACCACTTGCAATTA-3', and double-stranded DNA (shRNA) was synthesized (Invitrogen Inc., Carlsbad, CA, USA). The shRNA was recombined into the lentivirus plasmid pGIPZ (Molecular Biology Laboratory of Shandong University, Shandong, China), and competent DH5 $\alpha$ cells (Solarbio, Shanghai, China) were transformed.

Table 1. The primer sequences for qRT-PCR. Note: qRT-PCR, quantitative real-time polymerase chain reaction; GAPDH, Glyceraldehyde-3-phosphate dehydrogenase; PK, pyruvate kinase; F, forward; R, reverse

\begin{tabular}{ll}
\hline Gene & Primer sequences \\
\hline \multirow{2}{*}{ PKM2 } & F: 5'-GGAGCGAGA TCCC TCCAAAAT-3' \\
& R: 3'-GGCTGTTGTCATACTTCTCATGG-5' \\
p53 & F: 5'-ATGAACCGCCGACCTATC-3' \\
& R: 3'-TGGGCAATCCTTTAACTCTAA-5' \\
p21 & F: 5'-GCACTCAGAGGAGGCGCCATGTCA-3' \\
& R: 3'-CTGTCCCCTGCAGCAGAGCAGG -5' \\
GAPDH & F: 5'-GGAGCGAGA TCCC TCCAAAATT-3' \\
& R: 3'-GGCTGTTGTCATACTTCTCATGG-5' \\
\hline
\end{tabular}
Targeted cells were selected for recombinant cloning and identified by PCR with sequencing verification. Recombinant plasmids from the correctly sequenced cells were extracted, and calcium phosphate was used to package 293T cells provided by the molecular biology laboratory of Shandong University (Shandong, China). Viral supernatant was collected after $48 \mathrm{~h}$. Microporous membrane filtration and ultracentrifugation were successively performed, and the virus precipitation was resuspended in ice-cold phosphate buffered saline (PBS). The acquired shRNA lentivirus particles of PKM2 were referred to as PKM2-shRNA.

\section{Cell Grouping and Transfection}

LS-147T and SW620 cells in logarithmic phase were selected and cultured in 6-well plates, and transfection was prepared when cell fusion reached 70\%. Both cells were grouped into blank (no transfection), PKM2-shRNA (transfection with shRNA) and empty plasmid (transfection with empty plasmid) groups, and PKM2-shRNA and PKM2-NC viruses were added to the PKM2-shRNA and empty plasmid groups with the addition of $8 \mu \mathrm{g} / \mathrm{mL}$ polybrene (Solarbio, Shanghai, China). Meanwhile, an equal volume of medium was added to the blank group. Fresh medium was the substitute when cells were cultured for $24 \mathrm{~h}$, and green fluorescent protein (GFP) expression at $48 \mathrm{~h}$ was observed using a microscope. After the medium was changed, LS-147T-PKM2-shRNA, LS-147T-PKM2-NC SW620-PKM2-shRNA and SW620-PKM2-NC cells were obtained.

\section{Immunofluorescence}

LS-147T and SW620 cells in logarithmic phase were inoculated in 24-well plates with slides, and 4\% paraformaldehyde (Solarbio, Shanghai, China) was added to immobilize cells for 10 min when cell fusion achieved 90\%. Blocking solution containing 5\% FBS and 0.25\% Triton X-100 (Solarbio, Shanghai, China) was utilized to block the culture plates for $30 \mathrm{~min}$. A primary antibody for rabbit anti-human PKM2 (MAB8733, Solarbio, Shanghai, China) diluted using blocking solution at a ratio of 1:100 was added. The cell cultures were placed at $4^{\circ} \mathrm{C}$ overnight followed by rinsing in Tris-buffered saline tween (TBST) in triplicate for 5 min. The cell cultures were then mixed with horseradish peroxidase-labeled goat anti-rabbit secondary antibody (MB005, Solarbio, Shanghai, China) diluted as indicated above. The samples were incubated at room temperature for $1 \mathrm{~h}$ and stained with 4', 6-diamidino-2-phenylindole (DAPI) for $10 \mathrm{~min}$ in the dark. After glycerin fixation, cells were observed and photographed under a fluorescence microscope. Every test was run in triplicate.

\section{Western Blotting}

Total cellular proteins were extracted from cells after $48 \mathrm{~h}$ with transfection. Protein concentrations were determined using BCA kits (Solarbio, Shanghai, China). The extracted proteins with loading buffer were boiled at $95^{\circ} \mathrm{C}$ for $10 \mathrm{~min}$, with $30 \mu \mathrm{g}$ of sample loaded in each well, $10 \%$ polyacrylamide gel was used to electrophoretic separation protein (SDS-PAGE) (Solarbio, Shanghai, China). Electrophoresis voltage was tuned $80 \mathrm{~V}$ to $120 \mathrm{~V}$. Wet membrane [polyvinylidene fluoride (PVDF) membrane] transfer was conducted at a voltage of $100 \mathrm{mv}$ for 45 to $70 \mathrm{~min}$. Afterwards, the membranes were blocked at room temperature 


\section{Cellular Physiology Cell Physiol Biochem 2017;42:1769-1778 \begin{tabular}{l|l} 
DOI: 10.1159/000479456 & and Biochemistry \\
Published online: July 26, 2017 & $\begin{array}{l}\text { 2017 The Author(s). Published by S. Karger AG, Basel } \\
\text { www.karger.com/cpb }\end{array}$
\end{tabular} \\ Ao et al.: PKM2 and LS-147T and SW620 Cell Apoptosis and Proliferation}

for $1 \mathrm{~h}$ with $5 \%$ bovine serum albumin (BSA) (Solarbio, Shanghai, China) and then incubated with the following primary antibodies overnight at $4^{\circ} \mathrm{C}$ : rabbit anti-human PKM2 (MAB8733, 1: 1000, Solarbio, Shanghai, China), rabbit anti-human p53 (K000465P, 1:500, Solarbio, Shanghai, China), rabbit anti-human p53 (orb11203, 1:1000, Biorbyt Ltd., Hubei, China) and rabbit anti-human GAPDH (AG019, 1:1000, Solarbio, Shanghai, China). After incubation, the membranes were rinsed with TBST in triplicate for 5 min and then incubated with horseradish peroxidase-labeled goat anti-rabbit secondary antibody (MB005, Solarbio, Shanghai, China) (1:5000) at room temperature for $1 \mathrm{~h}$. Then, the membranes were washed with TBST as previously described. Finally, the membranes were visualized with chemiluminescence. Bio-Rad Gel Doc EZ Imager (Bio-Rad, California, USA) was used for imaging, and target bands were analyzed for gray level analysis using ImageJ (Bio-Rad, California, USA).

\section{Cell Counting Kit-8 (CCK-8) Assay}

After 48-h transfection, the cell suspension was collected and inoculated in 96-well plates in $100 \mu \mathrm{L}$ per well. The culture plates were placed in a $37^{\circ} \mathrm{C}$ incubator, and 3 wells were chosen randomly at 12,24 , 36, 48 and $72 \mathrm{~h}$ among three groups, separately. The cells in the selected wells were incubated with $10 \mu \mathrm{L}$ of CCK-8 for an additional $2 \mathrm{~h}$. A microplate reader (Bio-Rad, California, USA) was applied to detect the optical density (OD) of each well at a wavelength of $450 \mathrm{~nm}$ to generate a cell growth curve. Every test was performed in triplicate.

\section{Flow Cytometry}

Targeted cells were digested with $0.25 \%$ trypsin, and $1 \times 10^{6}$ cells were immobilized with $75 \%$ cold ethanol (Solarbio, Shanghai, China) for $12 \mathrm{~h}$. Cells were incubated with $50 \mu \mathrm{g} / \mathrm{mL}$ RNA enzymes (Solarbio, Shanghai, China) at room temperature for $1 \mathrm{~h}$ followed by propidium iodide (PI) (Solarbio, Shanghai, China) staining for $30 \mathrm{~min}$ in the dark. Flow cytometry (Bio-Rad, California, USA) was used to analyze the cell cycle. Every test was performed in triplicate.

The harvested cells were counted, and $1 \times 10^{6}$ cells were mixed with Annexin V (Solarbio, Shanghai, China) labeled with fluorescein isothiocyanate (FITC) (Solarbio, Shanghai, China) and incubated at $37^{\circ} \mathrm{C}$ for $10 \mathrm{~min}$. Following three PBS washes for $5 \mathrm{~min}$, PI staining was conducted in the dark at room temperature for $15 \mathrm{~min}$, and cell apoptosis was detected by flow cytometry. The apoptosis rate is presented as the percentage of early apoptotic cells among total cells. Every test was performed in triplicate.

\section{Senescence-associated $\beta$-galactosidase Staining}

After 48-h cell transfection, a senescence-associated $\beta$-galactosidase staining kit was used to assess cell senescence among all groups. The cell suspension was seeded in a 24-well plate, and the cell culture solution was removed followed by PBS washing. One milliliter of senescence-associated $\beta$-galactosidase staining fixing solution was used to fix for $15 \mathrm{~min}$ and was then aspirated. PBS solution was used for three washes ( $3 \mathrm{~min} /$ time) and also aspirated. Then, $1 \mathrm{~mL}$ of working solution was added to each well and incubated at $37^{\circ} \mathrm{C}$ overnight. The senescent cells appeared blue under an inverted microscope.

\section{Statistical Analysis}

The statistical analyses were conducted with SPSS 21.0 software (Shanghai Kabé Information Technology Co. Ltd. Shanghai, China). All measurement data were presented as $\bar{x} \pm \mathrm{s}$. Means of two samples were analyzed using $t$-tests. Differences between groups were analyzed using one-way analysis of variance (ANOVA) (with variance homogeneity performed previously). Pairwise comparisons among multiple sets of data were analyzed using the least significant difference (LSD) test. $P<0.05$ was considered statistically significant.

\section{Results}

LS-147T and SW620 Cells were Selected for Cell Transfection in Vitro

qRT-PCR results were presented in Fig. 1. PKM2 mRNA expression among six CRC LS147T cells exhibited the following order: LS-147T > SW620 > HT29 > HCT116 > SW480 > DLD1. The highest expression was noted in LS-147T and SW620 cell lines. Thus, the LS- 


\section{Cellular Physiology Cell Physiol Biochem 2017;42:1769-1778 \begin{tabular}{l|l} 
DOI: 10.1159/000479456 & O 2017 The Author(s). Published by S. Karger AG, Basel \\
www.karger.com/cpb
\end{tabular}

147T and SW620 cell lines were selected for further use.

LS-147T and SW620 Cells were Successfully Infected by Lentivirus

The expression vector pGIPZ utilized in the study carried GFP; thus, fluorescence intensity could be observed to determine transfection efficiency. In these two cell lines, fluorescence microscopy revealed no fluorescence in the blank group, whereas greater than 95\% GFP expression was noted in both the PKM2-shRNA and empty plasmid groups (Fig. 2). These results indicated that the lentivirus infection rate was greater than 95\% in CRC LS-147T and SW620 cells.

Comparisons of mRNA and Protein Expression of PKM2, p53 and p21 in CRC LS-147T Cells among the Blank, Empty Vector and PKM2-shRNA Groups

Immunofluorescence revealed PKM2 protein (marked with red fluorescence) expression in the cytoplasm and nucleus (blue fluorescence with DAPI staining) in LS-147T and SW620 cells, suggesting that PKM2 protein was widely distributed in the cytoplasm and nucleus of CRC LS-147T and SW620 cells (Fig. 3A).

For qRT-PCR and Western blotting, PKM2 mRNA and protein expression in the PKM2-shRNA cells from both cell lines were significantly reduced, whereas $p 53$ and $p 21$ expression was increased compared with the empty plasmid and blank groups (all $P<$ 0.05 ). In addition, no significant differences in PKM2, p53 and p21 mRNA and protein expression were noted between the empty plasmid and blank groups $(P>0.05)$ (Fig. 3B and $3 C$ ). These results suggested that PKM2shRNA transfection suppressed PKM2 mRNA and protein expression in LS-147T and SW620 cells.

PKM2 Gene Silencing Inhibited CRC LS$147 T$ and SW620 Cell Proliferation

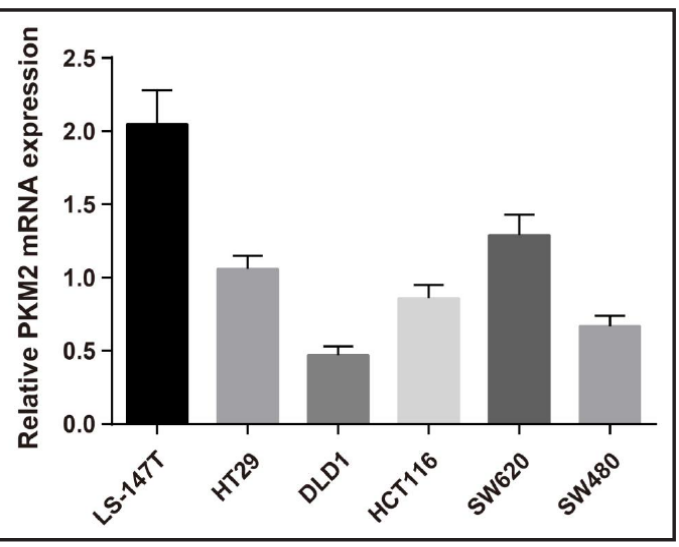

Fig. 1. PKM2 mRNA expression in CRC cell lines, including LS-147T, SW620, HT29, HCT116, SW480 and DLD1. Note: PK, pyruvate kinase; CRC, colorectal cancer.

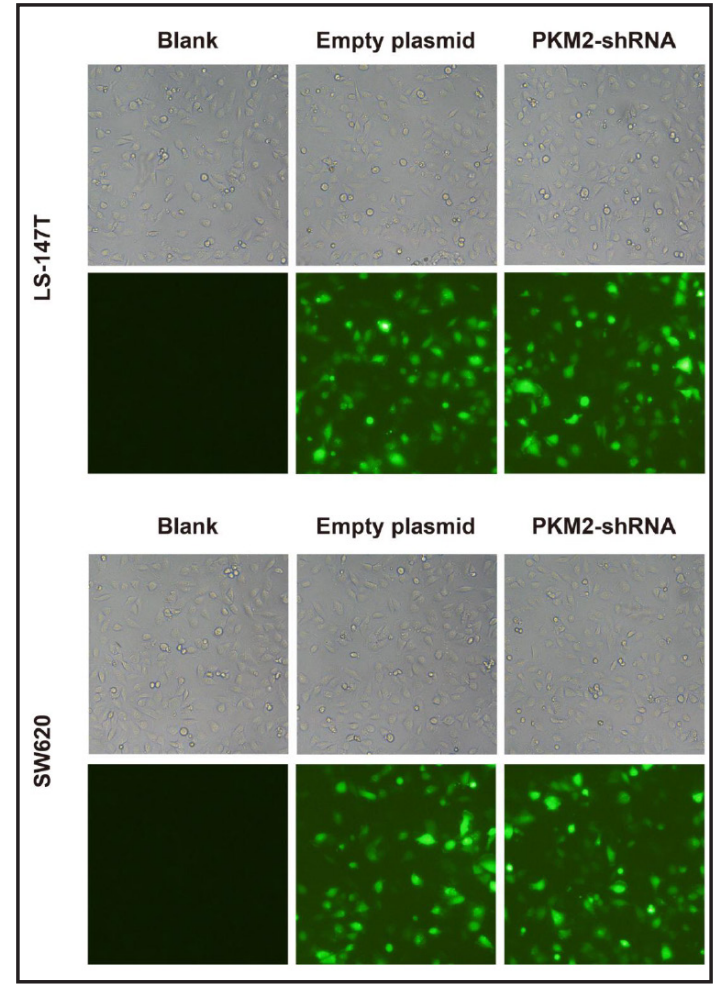

Fig. 2. Comparisons of GFP expression observed by fluorescence microscope $(\times 200)$ among the blank, empty vector and PKM2-shRNA groups. Note: GFP, green fluorescent protein.

Based on the CCK- 8 assay, cells in the

PKM2-shRNA group all exhibited markedly reduced OD values compared with the blank and empty plasmid groups at $12,24,36,48$ and $72 \mathrm{~h}$ (all $P<0.05$ ). When compared with the blank group, no significant differences in OD values were noted for the empty plasmid group $(P>0.05)$. For the cells within the group, cell proliferation enhanced with time (Fig. 4 ), indicating that PKM2 gene silencing evidently inhibited the proliferation of CRC LS-147T and SW620 cells. 


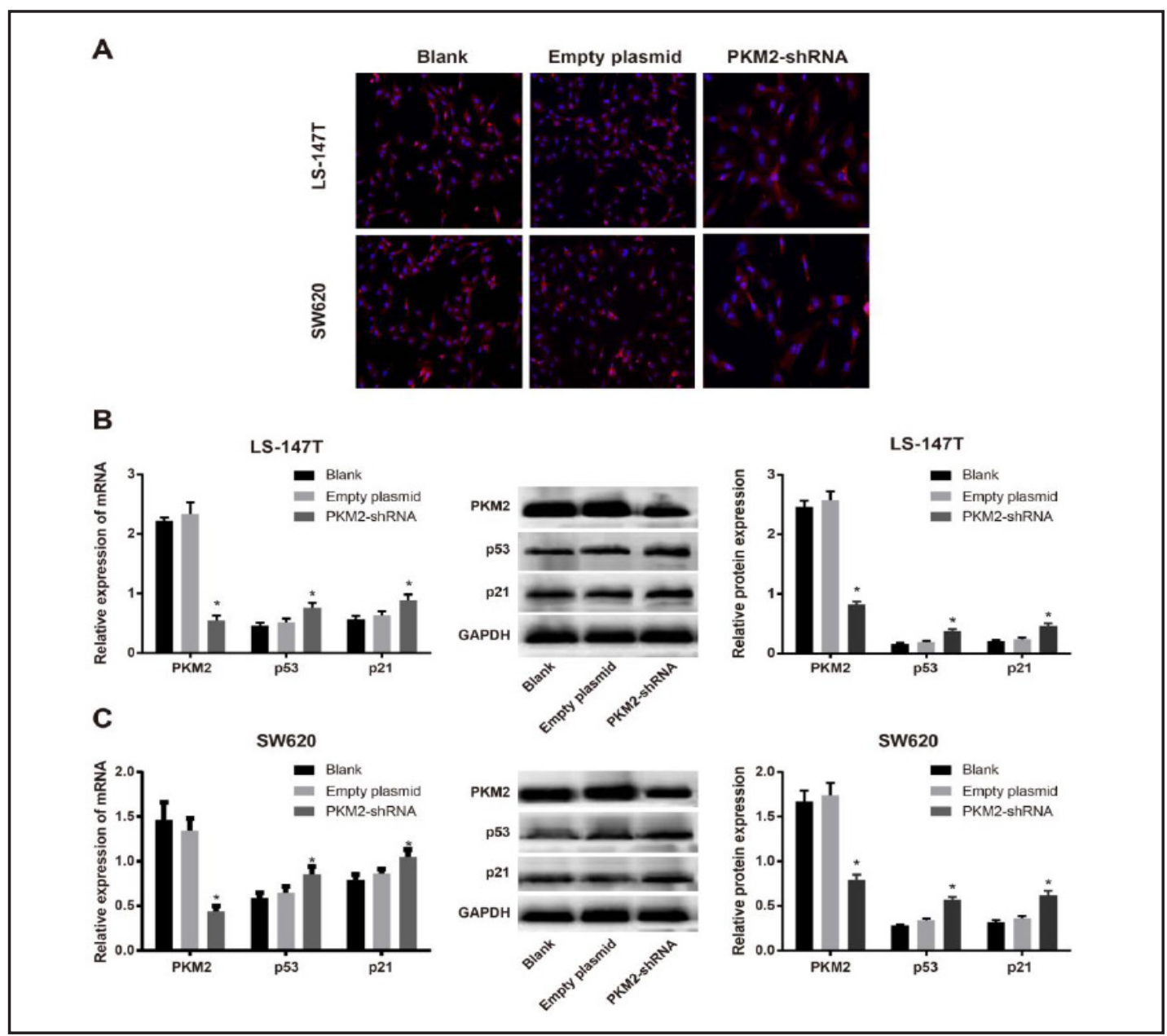

Fig. 3. Comparisons of PKM2, $p 53$ and $p 21 \mathrm{mRNA}$ and protein expression in LS-147T and SW620 cells in the blank, empty vector and PKM2-shRNA groups. Note: (A) PKM2 protein expression in LS-147T and SW620 cells detected by immunofluorescence ( $\times 200$ ); B1, PKM2, p53 and p21 mRNA and protein expression in LS$147 \mathrm{~T}$ cells detected by qRT-PCR; B2, Band and statistical analysis images of PKM2, p53 and p21 mRNA and protein expression in LS-147T cells detected by Western blotting; C1, PKM2, p53 and p21 mRNA expression in SW620 cells detected by qRT-PCR; C2, Bands and relative expression of PKM2, p53 and p21 proteins in SW629 cells detected by Western blotting; ${ }^{*}, P<0.05$ compared with the empty plasmid and blank groups, $\mathrm{n}=3$; PK, pyruvate kinase; qRT-PCR, quantitative real-time polymerase chain reaction.

\section{PKM2 Gene Silencing Reduced CRC LS-147T and SW620 Cell Replication}

Flow cytometry proved that in these two cell lines, the number of cells in the $\mathrm{G}_{1}$ phase of the PKM2-shRNA group distinctively increased in contrast with the blank group and empty plasmid group (all $P<0.05$ ). Simultaneously, a reduced cell ratio in the $\mathrm{G}_{2} / \mathrm{M}$ phase was noted in the PKM2-shRNA group compared with the blank and empty plasmid groups (all $P<0.05$ ). Moreover, no significant differences were noted in each phase between the blank group and empty plasmid groups (all $P>0.05$ ) (Table 2, Fig. 5). When PKM2 was silenced, CRC LS-147T and SW620 cell cycle progression was suppressed, and the $\mathrm{G}_{1}$ phase was inhibited, reducing cell replication.

PKM2 Gene Silencing promoted CRC LS-147T and SW620 Cell apoptosis

Cells were classified into 4 subgroups with the aid of flow cytometry. Among these groups, Annexin V-PI' represented normal cells, Annexin $\mathrm{V}^{+} / \mathrm{PI}^{-}$represented early apoptotic cells, Annexin $\mathrm{V}^{-} / \mathrm{PI}^{+}$represented cells with mechanical damage, and Annexin $\mathrm{V}^{+} / \mathrm{PI}^{+}$ represented late phase dead cells. In LS-147T cells, the apoptosis rate in the blank group was 
Fig. 4. CRC LS-147T and SW620 cell proliferation based on the CCK-8 assay among the blank, empty vector and PKM2-shRNA groups. Note: *, $P<0.05$ compared with empty plasmid and blank groups, $\mathrm{n}$ = 3; PK, pyruvate kinase; CRC, colorectal cancer; CCK, cell counting kit-8.

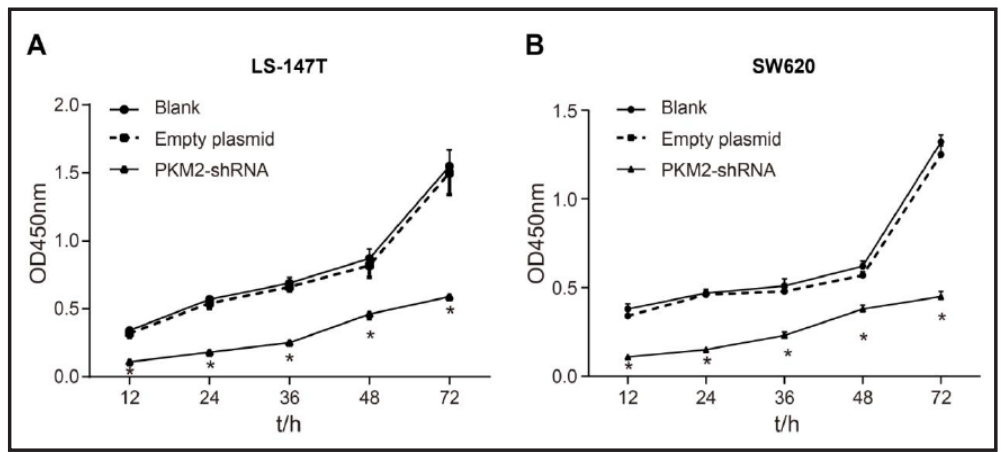

Fig. 5. The cell cycle in CRC LS-147T and SW620 cells as assessed by flow cytometry among the blank, empty vector and PKM2-shRNA groups. Note: PK, pyruvate kinase; CRC, colorectal cancer.

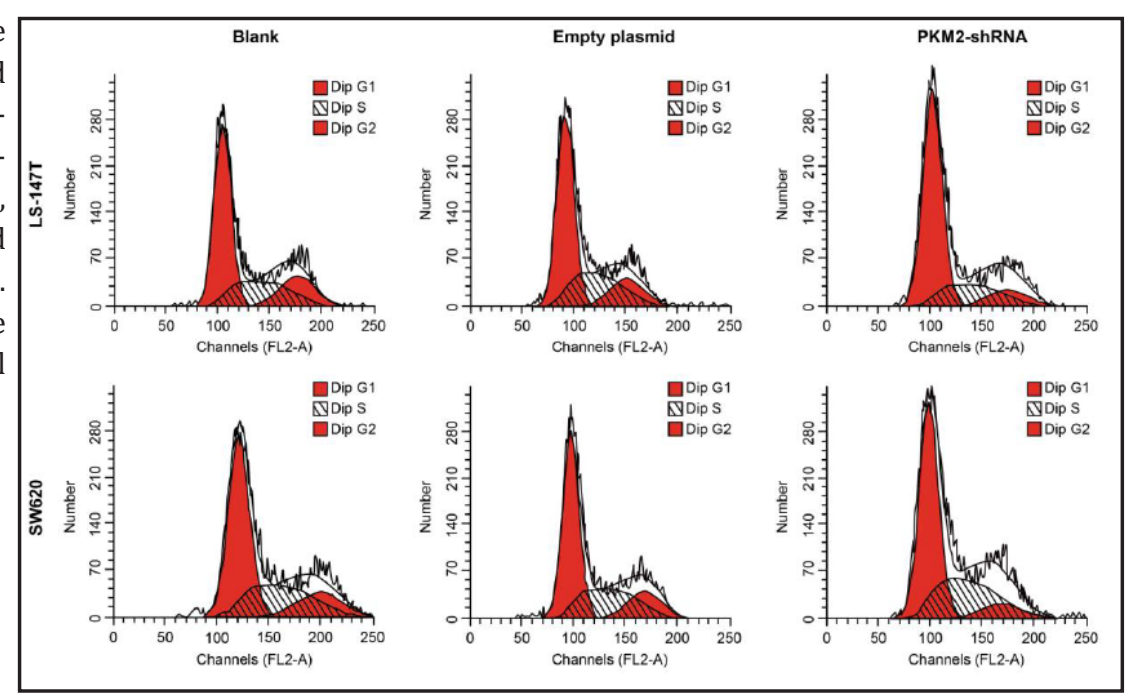

$(6.73 \pm 0.68) \%$, and the rate in the empty plasmid group was $(6.95 \pm 0.62) \%$. The PKM2-shRNA group exhibited a relatively increased apoptosis rate of (23.48 \pm $0.65) \%$. In SW620 cells, the apoptosis rate in the blank group was $(4.43 \pm 0.41) \%$, and the rate in the empty plasmid group was (4.65 \pm 0.52$) \%$. The PKM2-shRNA group exhibited an increased apoptosis rate of $(20.63 \pm 1.07) \%$. In general, the PKM2shRNA group exhibited a significantly higher apoptosis rate compared with the other two groups (all $P<0.05$ ), and no obvious differences were noted for the blank and empty plasmid groups $(P>$ 0.05 ) (Fig. 6). The above results suggested that PKM2 gene silencing markedly promotes CRC LS-147T and SW620 cell apoptosis.

\section{PKM2 Gene Silencing Accelerated CRC LS-147T and SW620 Cell Senescence}

As shown in Fig. 7, the blue granules were expressed as $\beta$-galactosidase, and the number of blue cells is indicative of the number of senescent cells. After PKM2 gene silencing, increased senescence-associated $\beta$-galactosidase activity was observed in the PKM2-shRNA group compared with the blank and empty plasmid groups. In LS-147T cells, the activity of the senescence marker $\beta$-galactosidase was $(0.52 \pm 0.09) \mathrm{mIU} / \mathrm{mL}$ and $(0.58 \pm 0.07) \mathrm{mIU} /$ $\mathrm{mL}$ in the blank and empty plasmid groups, respectively. The activity in the PKM2-shRNA group was $(2.17 \pm 0.22) \mathrm{mIU} / \mathrm{mL}$. In SW620 cells, the activity of the senescence marker 


\section{Cellular Physiology Cell Physiol Biochem 2017;42:1769-1778 \begin{tabular}{ll|l} 
DOI: 10.1159/000479456 & O 2017 The Author(s). Published by S. Karger AG, Basel \\
www.karger.com/cpb
\end{tabular}

Fig. 6. CRC LS-147T and SW620 cell apoptosis as assessed by flow cytometry among the blank, empty vector and PKM2-shRNA groups. Note: $\mathrm{PK}$, pyruvate kinase; CRC, colorectal cancer

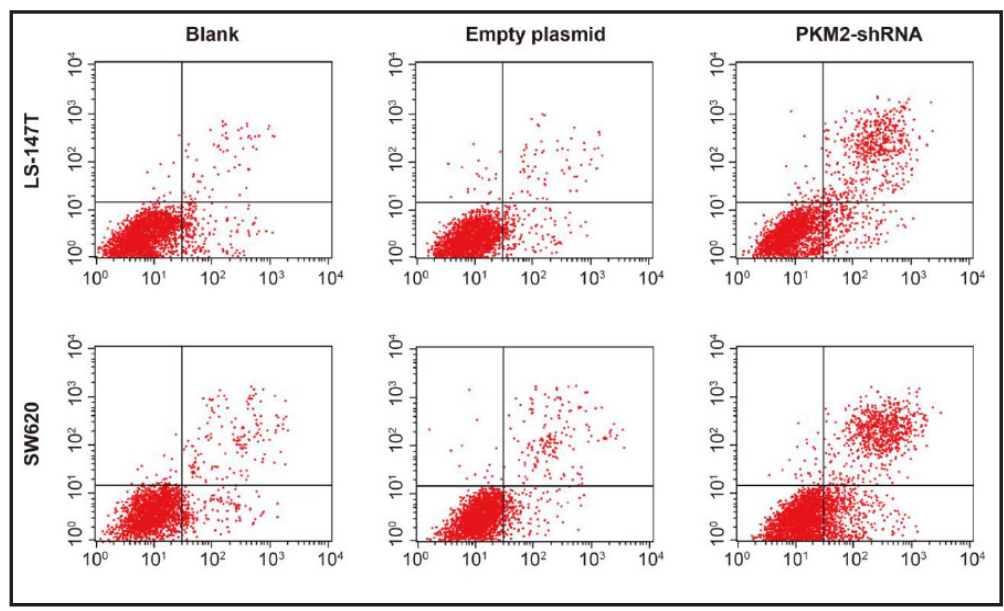

Fig. 7. Cell senescence in CRC LS$147 \mathrm{~T}$ and SW620 cells based on senescence-associated $\beta$-galactosidase staining among the blank, empty vector and PKM2-shRNA groups. Note: Comparison of $\beta$-galactosidase activity in LS-147T and SW620 cells; (B) The staining of senescent cells $(x$ 200); PK, pyruvate kinase; CRC, colorectal cancer; ${ }^{*}, P<0.05$ compared with the empty plasmid group and blank group.

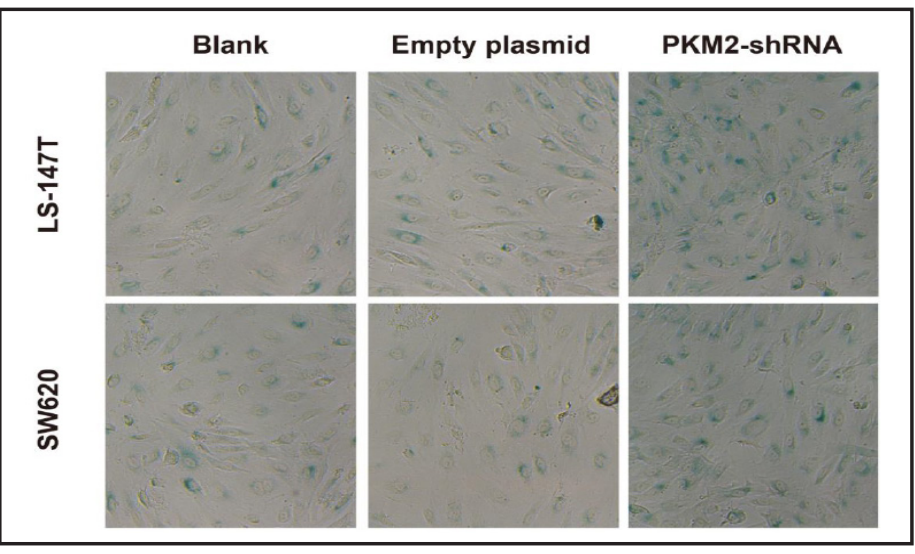

$\beta$-galactosidase was $(0.47 \pm 0.05) \mathrm{mIU} / \mathrm{mL}$ and $(0.43 \pm 0.05) \mathrm{mIU} / \mathrm{mL}$ in the blank and empty plasmid groups, respectively. The value in the PKM2-shRNA group was $(1.74 \pm 0.23) \mathrm{mIU} /$ $\mathrm{mL}$, which was significantly increased. However, no significant difference was noted between the blank and empty plasmid groups.

\section{Discussion}

CRC is widely viewed as one of the most common malignant tumors. Greater than one million people are diagnosed with CRC, and approximately half of them die from the malignancy each year. In addition, the CRC incidence is increasing yearly [20]. Therefore, available CRC markers are being pursued to diagnose tumor progression and discover effective therapeutic targets.

PKM2-shRNA transfection inhibits PKM2 mRNA and protein expression in CRC LS-147T and SW620 cells in this paper. RNA is typically induced by double-stranded RNA (dsRNA), and it can suppress the expression of target genes with high specificity [21]. RNA is a classical mechanism of gene expression regulation, it temporarily reduces active gene products via a process which known as gene knockdown, and it is different from gene knockout procedures [22]. Similarly, sequence-specific transcription induced by double-stranded RNA targeting PKM2 degrades mRNA homogenous to the PKM2 gene.

According to our findings, CRC LS-147T and SW620 cell cycle progression was suppressed, and cells were inhibited in $\mathrm{G}_{1}$ phase when PKM2 gene silencing occurred, leading to reduced cell replication capacity. However, in addition to the fact that PKM2 is a crucial regulator in glycolysis and provides energy for tumor cells under conditions of transformation between highly active tetramer and inactive dimer, PKM2 also plays important roles in regulating the 


\section{Cellular Physiology Cell Physiol Biochem 2017;42:1769-1778

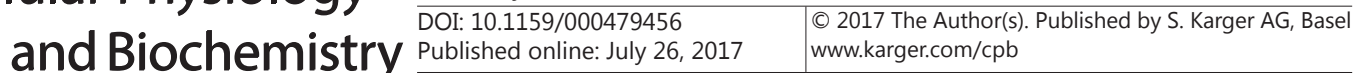 \\ Ao et al.: PKM2 and LS-147T and SW620 Cell Apoptosis and Proliferation}

cell cycle and promoting cell proliferation [23]. We demonstrated that DNA proliferation was inhibited in the $\mathrm{G}_{1}$ phase in CRC LS-147T and SW620 cells, and cells in the $\mathrm{G}_{2} / \mathrm{M}$ phase were obviously reduced due to insufficient levels of synthetic substrates required for cancer cell proliferation when the PKM2 gene was silenced, further inhibiting cancer cell replication.

PKM2 gene silencing suppresses CRC LS-147T and SW620 cell proliferation and accelerates apoptosis. It should be noted that mitochondrial respiration persists in the vast majority of tumors and remains a major source for ATP generation. In addition, a profoundly elevated flux of glucose intake is observed for lactate fermentation to produce energy via a process called the "Warburg Effect", which increases glucose consumption compared with normal cells and leads to tumor cell immortalization [24, 25]. Previous studies also confirmed that aerobic glycolysis under PKM2 regulation causes cancer cell proliferation in a hypoxic tumor environment. In addition, the "Warburg Effect" in tumor cells is constantly enhanced by the conversion of dimers and tetramers of PKM2 to provide energy for infinite proliferation of tumor cells $[8,26,27]$. In addition, mitochondria, which are recognized as an important organelle for ATP generation and energy release in animal cells, are directly associated with apoptosis, and mitochondrial membrane potential (MMP) is generated due to the asymmetric distribution of proteins on the concentration gradient across the inner mitochondrial membrane in respiratory oxidation processes. In contrast, normal MMP is a prerequisite for the cellular capacity to generate ATP by oxidative phosphorylation [28]. Moreover, the structure of mitochondrial permeability transition pore (MPT), which is related to apoptosis and necrotic cell death in mitochondrial adventitia, is damaged when PKM2 expression is suppressed, hindering the facilitation of a mass exchange between the inner and outer membrane of mitochondria and resulting in rapid loss of MMP and tumor cell apoptosis [29].

In conclusion, transfection of PKM2-shRNA can effectively reduce PKM2 expression in CRC LS-147T and SW620 cells, and PKM2 gene silencing impedes LS-147T and SW620 cell proliferation and the cell cycle and promotes cell apoptosis. Therefore, PKM2 gene silencing is a promising and effective CRC therapeutic approach; however, further investigations about the specific mechanisms are warranted.

\section{Acknowledgments}

We would like to acknowledge the helpful comments on this paper received from our reviewers.

\section{Disclosure Statement}

None.

\section{References}

1 Yan S, Cao Y, Mao A: MicroRNAs in colorectal cancer: potential biomarkers and therapeutic targets. Front Biosci (Landmark Ed) 2015;20:1092-1103.

2 Jemal A, Bray F, Center MM, Ferlay J, Ward E, Forman D: Global cancer statistics. CA Cancer J Clin 2011;61:69-90.

-3 Bhalla A, Zulfiqar M, Weindel M, Shidham VB: Molecular diagnostics in colorectal carcinoma. Clin Lab Med 2013;33:835-859.

4 Courtney RJ, Paul CL, Carey ML, Sanson-Fisher RW, Macrae FA, D'Este C, Hill D, Barker D, Simmons J: A population-based cross-sectional study of colorectal cancer screening practices of first-degree relatives of colorectal cancer patients. BMC Cancer 2013;13:13.

5 Dahan L, Norguet E, Etienne-Grimaldi MC, Formento JL, Gasmi M, Nanni I, Gaudart J, Garcia S, Ouafik L, Seitz JF, Milano G: Pharmacogenetic profiling and cetuximab outcome in patients with advanced colorectal cancer. BMC Cancer 2011;11:496. 


\section{Cellular Physiology Cell Physiol Biochem 2017;42:1769-1778 \begin{tabular}{l|l} 
DOI: 10.1159/000479456 & Ond 2017 The Author(s). Published by S. Karger AG, Basel \\
www.karger.com/cpb
\end{tabular} \\ Ao et al.: PKM2 and LS-147T and SW620 Cell Apoptosis and Proliferation}

6 Mik M, Dziki L, Trzcinski R, Dziki A: Risk factors of 30-day mortality following surgery for colorectal cancer. Pol Przegl Chir 2016;88:26-31.

7 Berger AM, Grem JL, Visovsky C, Marunda HA, Yurkovich JM: Fatigue and other variables during adjuvant chemotherapy for colon and rectal cancer. Oncol Nurs Forum 2010;37:E359-369.

8 Zhou CF, Li XB, Sun H, Zhang B, Han YS, Jiang Y, Zhuang QL, Fang J, Wu GH: Pyruvate kinase type M2 is upregulated in colorectal cancer and promotes proliferation and migration of colon cancer cells. IUBMB Life 2012;64:775-782.

-9 Li Z, Yang P, Li Z: The multifaceted regulation and functions of PKM2 in tumor progression. Biochim Biophys Acta 2014;1846:285-296.

10 Mazurek S: Pyruvate kinase type M2: a key regulator of the metabolic budget system in tumor cells. Int J Biochem Cell Biol 2011;43:969-980.

11 Yang W, Xia Y, Hawke D, Li X, Liang J, Xing D, Aldape K, Hunter T, Alfred Yung WK, Lu Z: PKM2 phosphorylates histone $\mathrm{H} 3$ and promotes gene transcription and tumorigenesis. Cell 2012;150:685-696.

12 Mukherjee J, Ohba S, See WL, Phillips JJ, Molinaro AM, Pieper RO: PKM2 uses control of HuR localization to regulate p27 and cell cycle progression in human glioblastoma cells. Int J Cancer 2016;139:99-111.

13 Wong N, Yan J, Ojo D, De Melo J, Cutz JC, Tang D: Changes in PKM2 associate with prostate cancer progression. Cancer Invest 2014;32:330-338.

14 Shashni B, Sakharkar KR, Nagasaki Y, Sakharkar MK: Glycolytic enzymes PGK1 and PKM2 as novel transcriptional targets of PPARgamma in breast cancer pathophysiology. J Drug Target 2013;21:161-174.

15 Yu G, Yu W, Jin G, Xu D, Chen Y, Xia T, Yu A, Fang W, Zhang X, Li Z, Xie K: PKM2 regulates neural invasion of and predicts poor prognosis for human hilar cholangiocarcinoma. Mol Cancer 2015;14:193.

16 Maduri S: Applicability of RNA interference in cancer therapy: Current status. Indian J Cancer 2015;52:1121.

-17 Liu J, Wen D, Fang X, Wang X, Liu T, Zhu J: p38MAPK Signaling Enhances Glycolysis Through the UpRegulation of the Glucose Transporter GLUT-4 in Gastric Cancer Cells. Cell Physiol Biochem 2015;36:155165.

18 Chen S, Li P, Li J, Wang Y, Du Y, Chen X, Zang W, Wang H, Chu H, Zhao G, Zhang G: MiR-144 inhibits proliferation and induces apoptosis and autophagy in lung cancer cells by targeting TIGAR. Cell Physiol Biochem 2015;35:997-1007.

19 Tuo YL, Li XM, Luo J: Long noncoding RNA UCA1 modulates breast cancer cell growth and apoptosis through decreasing tumor suppressive miR-143. Eur Rev Med Pharmacol Sci 2015;19:3403-3411.

20 Jimenez CR, Knol JC, Meijer GA, Fijneman RJ: Proteomics of colorectal cancer: overview of discovery studies and identification of commonly identified cancer-associated proteins and candidate CRC serum markers. J Proteomics 2010;73:1873-1895.

21 Wang J, Mi P, Lin G, Wang YX, Liu G, Chen X: Imaging-guided delivery of RNAi for anticancer treatment. Adv Drug Deliv Rev 2016;104:44-60.

22 Gottumukkala SN, Dwarakanath CD, Sudarsan S: Ribonucleic acid interference induced gene knockdown. J Indian Soc Periodontol 2013;17:417-422.

-23 Gao X, Wang H, Yang JJ, Liu X, Liu ZR: Pyruvate kinase M2 regulates gene transcription by acting as a protein kinase. Mol Cell 2012;45:598-609.

24 Lu J, Tan M, Cai Q: The Warburg effect in tumor progression: mitochondrial oxidative metabolism as an anti-metastasis mechanism. Cancer Lett 2015;356:156-164.

25 Yecies JL, Manning BD: Chewing the fat on tumor cell metabolism. Cell 2010;140:28-30.

26 Chaneton B, Gottlieb E: Rocking cell metabolism: revised functions of the key glycolytic regulator PKM2 in cancer. Trends Biochem Sci 2012;37:309-316.

27 Yang W, Zheng Y, Xia Y, Ji H, Chen X, Guo F, Lyssiotis CA, Aldape K, Cantley LC, Lu Z: ERK1/2-dependent phosphorylation and nuclear translocation of PKM2 promotes the Warburg effect. Nat Cell Biol 2012;14:1295-1304.

28 Perry SW, Norman JP, Barbieri J, Brown EB, Gelbard HA: Mitochondrial membrane potential probes and the proton gradient: a practical usage guide. Biotechniques 2011;50:98-115.

-29 Bonora M, Pinton P: The mitochondrial permeability transition pore and cancer: molecular mechanisms involved in cell death. Front Oncol 2014;4:302. 